\title{
Generalised hyperbolicity in spacetimes with Lipschitz regularity.
}

\author{
Yafet Sanchez Sanchez* \\ and James A. Vickers ${ }^{\dagger}$ \\ Mathematical Sciences and STAG Research Centre, \\ University of Southampton, \\ Southampton, \\ SO17 1BJ
}

October 12, 2016

\begin{abstract}
In this paper we obtain general conditions under which the wave equation is well-posed in spacetimes with metrics of Lipschitz regularity. In particular, the results can be applied to spacetimes where there is a loss of regularity on a hypersurface such as shell-crossing singularities, thin shells of matter and surface layers. This provides a framework for regarding gravitational singularities not as obstructions to the world lines of point-particles, but rather as obstruction to the dynamics of test fields.
\end{abstract}

\section{Introduction}

An important requirement of any classical physical theory is that given suitable initial data one can determine the evolution of the system. Within the theory of general relativity the concept of global hyperbolicity [1] therefore plays a key role. Mathematically, a spacetime region $\mathcal{N}$ is said to globally hyperbolic if the causality condition is satisfied, and for any two points $p, q \in \mathcal{N}$ the causal diamond $J^{+}(p) \cap J^{-}(q)$ is compact and contained in $\mathcal{N}$ [2]. However from the physical point of view the important property of smooth globally hyperbolic spacetimes is that the evolution of the Einstein equations is well defined (see e.g. 3] for details).

One way of interpreting the compactness of $J^{+}(p) \cap J^{-}(q)$ is that this set "does not contain any points on the edge of spacetime, i.e. at infinity or at a singularity" [3, §6.6]. In this context the regularity usually considered in the

\footnotetext{
*E-mail:Y.SanchezSanchez@soton.ac.uk

${ }^{\dagger}$ E-mail:J.A.Vickers@soton.ac.uk
} 
region $\mathcal{N}$ is that the metric must be $C^{1,1}$ (also denoted $\left.C^{2-}\right) 1$. This criteria is based on the existence and uniqueness of geodesics and the characterisation of gravitational singularities in terms of geodesic incompleteness. Moreover this regularity is the threshold where causal theory for smooth metrics and rough metrics agree [4, 5].

However, in many realistic astrophysical situations one would like to solve Einstein's Field Equations with lower regularity. For example, when one models a jump in density at the boundary of a star. This led Clarke [6] to define a notion of generalised hyperbolicity directly in terms of the local existence and uniqueness of the wave equation.

Clarke's definition of generalised hyperbolicity was motivated by two things. Firstly there are a number of spacetimes in which points are removed due to the presence of weak singularities and are therefore not globally hyperbolic but are still of physical interest. These include spacetimes with thin shells of matter [7] , impulsive gravitational waves [8] and shell-crossing singularities [9]. The second motivation was that of using test fields (given by solutions of the wave equation) rather than test particles (given by solutions of the geodesic equation) to probe the structure of singularities. Some work to examine the physical effect of gravitational singularities has been done by using a 3-parameter family of test particles (see for example [10]), however an advantage of using test fields is that the behaviour of the naturally defined energy-momentum tensor of the field gives a direct measurement of the physical effect of the singularity which is not so easy when considering families of test particles.

There have been previous approaches which study the nature of the singularities using test fields (see e.g. [11, 12, 13, 14] ). In all these approaches one considers self-adjoint extensions of the (spatial) Laplace-Beltrami operator and applies appropriate boundary conditions at the singularity. All this work has focused on the case of static spacetimes and furthermore most of these self-adjoint extensions are in $L^{2}$ (except [14] who consider finite energy solutions).

The approach in this paper is to consider singularities as interior points of a spacetime with low regularity rather than regarding the singularity as a boundary and applying boundary conditions. A natural condition in this context is to require the solutions to lie in the Sobolev space $H_{l o c}^{1}$ which ensures that the energy momentum tensor is well-defined as a distribution. In this paper we will therefore look at conditions on the spacetime and initial data which give solutions with this regularity.

In particular, we will look at the co-dimension one case which is relevant for example to junction conditions with jumps in the density, impulsive

\footnotetext{
${ }^{1}$ A function $f$ on an open set $\mathcal{U}$ of $\mathbb{R}^{n}$ is said to be Lipschitz or $C^{0,1}$ if there is some constant $K$ such that for each pair of points $p, q \in \mathcal{U},|f(p)-f(q)| \leqslant K|p-q|$, where $|p|$ denotes the usual Euclidean distance. We denote by $C^{k, 1}$ those functions where the $k$ th derivative is a Lipschitz function.
} 
gravitational waves and brane-world cosmologies. In a previous paper we looked at the case of co-dimension two singularities such as cosmic strings [15].

In previous work Grant et al [16] showed the existence of generalised solutions to the wave equation on singular spacetimes. This involved replacing the singular metric by a 1-parameter family of smooth metrics. The 1-parameter family of solutions of the corresponding wave equations then describes a generalised solution. Using this approach it was possible to demonstrate the existence of a generalised solution to the wave equation for a wide class of metrics which included metrics with components bounded almost everywhere. A downside of this approach is that it is not easy to relate the generalised solution to a classical weak solution of the equation. In our approach we will utilise the vanishing viscosity method (see e.g. Evans [17]) to show the existence of a weak solution of the wave equation. This involves approximating the wave equation by a system of second order parabolic equations with a parameter $\epsilon$ corresponding to the viscosity. Like Grant et al we again obtain a 1-parameter family of solutions but we are able to utilise results from parabolic regularity theory to have better control over the solutions and their convergence. This enables us to show the 1-parameter family converges to a weak solution of the zero viscosity equation which corresponds to an $H^{1}$ solution of the wave equation. The basic method we use follows that of Evans [17] but the details differ and our results are also distinct from from his since we assume less regularity and as a result our solutions also exhibit less regularity.

The plan of the paper is as follows. In $\S 2$ we introduce the general setting for the problem and state the main theorems. We do not impose the ultrastatic condition used in [18] and write the wave equation as a first order system (see e.g. [19]). This enable us to work with the $L^{2}$ energy of the first order system which corresponds to an $H^{1}$ energy of the second order system. In this setting we obtain existence of the solutions in time dependent scenarios without the need for any symmetries. $\S 3$ contains the proofs of the theorems while $\S 4$ gives examples of how these can be applied to various examples such as a discontinuity across a hypersurface, impulsive gravitational waves and brane-world cosmologies.

\section{The main results}

The interest in co-dimension one singular submanifolds covers a variety of different interesting physical phenomena such as, surface layers [7, impulsive gravitational waves [8], and shell-crossing singularities [20] all of which fall outside the class of smooth globally hyperbolic spacetimes. Moreover, the mathematical analysis by Geroch and Traschen [21] of what now is called the class of Geroch-Traschen metrics and the subsequent analysis by Stein- 
bauer and Vickers [22] using generalised functions gives co-dimension one singular submanifolds a robust mathematical background. In addition, recent proposals in semi-classical gravity and quantum gravity [23] suggest that the metric near the event horizon may present some loss of regularity. In this section we present techniques to prove local well-posedness of the wave equation in spacetimes with co-dimension one singularities subject to certain condition on the metric.

Clarke used the term generalised hyperbolicity to describe the situation where one has a unique solution to the wave equation. However we want to impose the slightly stronger condition of well-posedness and in addition want to emphasise the role of the wave equation. See [24] for a discussion of this and related terms.

\subsection{The general setting}

The geometric setting is a region $\Sigma_{[0, T]}=[0, T] \times \Sigma$, where $\Sigma$ is either a compact closed $n$-dimensional manifold or an open, bounded set of $\mathbb{R}^{n}$ with smooth boundary $\partial \Sigma$. In what follows, for simplicity, we will only consider the former case. The proof in the latter case follows by replacing $H^{1}(\Sigma)$ by $H_{0}^{1}(\Sigma)$ and using the volume form given by $d x^{n}$.

Rather than considering the particular case of a spacetime with a singular hypersurface, where the regularity of the metric drops below $C^{2}$, we will consider a rough spacetime, where the spacetime metric $g_{a b}$ is only Lipschitz. We will show that in this situation one has well-posedness (in a sense made precise below) of the wave equation with weak solutions of regularity $H^{1}\left(\Sigma_{[0, T]}\right)$. In order to do this, we will reformulate the wave equation as a first order symmetric hyperbolic system and look for $L^{2}$ solutions of this system.

We therefore start by considering the first order initial value problem

$$
\begin{aligned}
L \mathbf{u}=A^{0} \partial_{t} \mathbf{u}+A^{i} \partial_{i} \mathbf{u}+B \mathbf{u} & =\mathbf{F} \\
\mathbf{u}(0, \cdot) & =\mathbf{u}_{0}(\cdot)
\end{aligned}
$$

In the above we have employed the Einstein summation convention where $i$, $j, k$, etc. range over $1,2, \ldots, n$. The unknown $\mathbf{u}$ and the source term $\mathbf{F}$ are both $\mathbb{R}^{N}$ valued functions on $\Sigma_{[0, T]}$, while $A^{0}, A^{i}$ and $B$ are $N \times N$ matrix valued functions on $\Sigma_{[0, T]}$. We will assume that $A^{0}$ and $A^{i}$ are symmetric and that in addition $A^{0}$ is positive definite.

In order for such a system to correspond to the wave equation given by a Lipschitz metric, we will require that $A^{0}$ and $A^{i}$ have bounded first derivatives and that $B$ is bounded, so that we require

$$
A^{0} \in W^{1, \infty}\left(\Sigma_{[0, T]}, \mathbb{R}^{N^{2}}\right), \quad A^{i} \in W^{1, \infty}\left(\Sigma_{[0, T]}, \mathbb{R}^{N^{2}}\right), \quad B \in L^{\infty}\left(\Sigma_{[0, T]}, \mathbb{R}^{N^{2}}\right) .
$$


In the analysis below we will be working in spaces such as $L^{2}(\Sigma)$. Rather than defining this in terms of a particular coordinate system on $\Sigma$, we will introduce a background Riemannian metric $h_{i j}$ on $\Sigma$ and let $\nu_{h}$ be the corresponding volume form. We then define $L^{2}(\Sigma)$ to be the space of real valued functions $g$ on $\Sigma$ such that $\int_{\Sigma} g^{2} \nu_{h}<\infty$ and we denote the associated inner product by $(f, g)_{L^{2}(\Sigma)}=\int_{\Sigma} f g \nu_{h}$. Note that since $\Sigma$ is compact $\nu_{h}$ is bounded from below and above. Furthermore if $\Sigma$ is parallelizable (which for simplicity we will assume) there is no loss of generality in taking $h_{i j}$ to be the flat metric. Note that in the three dimensional case, which is most relevant to applications in general relativity, it is enough for $\Sigma$ to be orientable for it to be parallelizable.

For the case of vector valued functions $\mathbf{v}$ on $\Sigma$, we define $L^{2}\left(\Sigma, \mathbb{R}^{N}\right)$ to be those $\mathbf{v}$ such that $\int_{\Sigma} \mathbf{v} \cdot \mathbf{v} \nu_{h}<\infty$. The corresponding inner product on $L^{2}\left(\Sigma, \mathbb{R}^{N}\right)$ is then given by

$$
(\mathbf{v}, \mathbf{w})_{L^{2}\left(\Sigma, \mathbb{R}^{N}\right)}=\int_{\Sigma} \mathbf{v} \cdot \mathbf{w} \nu_{h}
$$

Where there is no risk of confusion, we will write both the inner product in $L^{2}(\Sigma)$ and in $L^{2}\left(\Sigma, \mathbb{R}^{N}\right)$ simply as $(\cdot, \cdot)_{L^{2}}$ The Sobolev spaces $H^{1}\left(\Sigma, \mathbb{R}^{N}\right)$ etc. are defined in a similar manner ( see[17] §5.2, [19]).

We also make use of the function space $L^{2}\left(\Sigma_{[0, T]}\right)$ which is defined by requiring that functions are square integrable on $[0, T] \times \Sigma$ with respect to the volume form $d t \wedge \nu_{h}$. However in the analysis below it is often convenient to think of a function $\mathbf{v}(t, x)$ as a map from $[0, T]$ to a function $\mathbf{v}(t)(\cdot)$ of $x \in \Sigma$ given by $\mathbf{v}(t)(x)=\mathbf{v}(t, x)$. For example $L^{2}\left(0, T ; L^{2}\left(\Sigma, \mathbb{R}^{N}\right)\right)$ is the space of functions

$$
\begin{aligned}
\mathbf{v}:[0, T] & \rightarrow L^{2}\left(\Sigma, \mathbb{R}^{N}\right) \\
t & \mapsto \mathbf{v}(t)
\end{aligned}
$$

such that $\mathbf{v}(t) \in L^{2}\left(\Sigma, \mathbb{R}^{N}\right)$ and

$$
\int_{0}^{T}(\mathbf{v}, \mathbf{v})_{L^{2}\left(\Sigma, \mathbb{R}^{N}\right)} d t<\infty
$$

When thinking of $\mathbf{v}$ in this way, we will denote the time derivative by $\dot{\mathbf{v}}$. We also define the following spaces which will be used in $§ 3.4$.

$$
\tilde{W}^{k, p}\left(\Sigma_{[0, T]}, \mathbb{R}^{N}\right):=\left\{\mathbf{w} \in W^{k, p}\left(\Sigma_{[0, T]}, \mathbb{R}^{N}\right): \text { such that } \mathbf{w}(T, \cdot)=0\right\}
$$

In particular, we will be interested in the following case

$$
\tilde{H}^{1}\left(\Sigma_{[0, T]}, \mathbb{R}^{N}\right):=\left\{\mathbf{w} \in H^{1}\left(\Sigma_{[0, T]}, \mathbb{R}^{N}\right): \text { such that } \mathbf{w}(T, \cdot)=0\right\} .
$$




\subsection{Weak solutions and the main theorems}

We will be looking for weak solutions of the initial value problem (1). To motivate the definition we proceed as follows. Given a standard $C^{1}$ solution $\mathbf{u}$ of the initial value problem, we may first take the dot product of equation (11) with a smooth $\mathbb{R}^{N}$-valued function $\mathbf{v}$ with support in $[0, T) \times \Sigma$ and then integrate over $x$ and $t$ to obtain

$$
\int_{0}^{T}(L \mathbf{u}, \mathbf{v})_{L^{2}\left(\Sigma, \mathbb{R}^{N}\right)} d t=\int_{0}^{T}(\mathbf{F}, \mathbf{v})_{L^{2}\left(\Sigma, \mathbb{R}^{N}\right)} d t
$$

Integrating the left hand side by parts with respect to $x$ and $t$ we obtain

$$
\int_{0}^{T}\left(\mathbf{u}, L^{*} \mathbf{v}\right)_{L^{2}\left(\Sigma, \mathbb{R}^{N}\right)} d t-\left(\left.A^{0} \mathbf{u}\right|_{t=0}, \mathbf{v}(0)\right)_{L^{2}\left(\Sigma, \mathbb{R}^{N}\right)}=\int_{0}^{T}(\mathbf{F}, \mathbf{v})_{L^{2}\left(\Sigma, \mathbb{R}^{N}\right)} d t
$$

where $L^{*}$ is the formal adjoint of $L$ defined below and the second term on the right hand side (RHS) comes from the $t=0$ boundary term when we integrate by parts with respect to $t$. This approach results in the following definition:

Definition 1 (Weak Solution) We say a function:

$$
\mathbf{u} \in L^{2}\left(0, T ; L^{2}\left(\Sigma, \mathbb{R}^{N}\right)\right)
$$

is a local weak solution of the initial value problem (1) provided that: For all $\mathbf{v} \in C^{\infty}\left(\Sigma_{[0, T]}, \mathbb{R}^{N}\right)$, with $\operatorname{supp}(\mathbf{v}) \subseteq[0, T) \times \Sigma$

$$
\int_{0}^{T}\left(\mathbf{u}, L^{*} \mathbf{v}\right)_{L^{2}\left(\Sigma, \mathbb{R}^{N}\right)} d t=\int_{0}^{T}(\mathbf{F}, \mathbf{v})_{L^{2}\left(\Sigma, \mathbb{R}^{N}\right)} d t+\left(A^{0}(0) \mathbf{u}_{0}, \mathbf{v}(0)\right)_{L^{2}\left(\Sigma, \mathbb{R}^{N}\right)} .
$$

This definition of a weak solution is the classical one used by Friedrichs [25] but differs from the one used by Evans [17, who does not integrate with respect to $t$. Note also that the formal adjoint is defined with respect to $\nu_{h}$. So in the case where we use a general Riemannian metric, there are additional terms involving the derivatives of $\nu_{h}$ in the expression for $L^{*}$ compared to the flat case. The explicit expression is:

$$
L^{*} \mathbf{v}:=-\partial_{t}\left(A^{0} \mathbf{v}\right)-\partial_{i}\left(A^{i} \mathbf{v}\right)+B^{T} \mathbf{v}-\tilde{\Gamma}_{l i}^{i} A^{l} \mathbf{v}
$$

where $\tilde{\Gamma}_{j k}^{i}$ are the connection coefficients of the smooth Riemannian metric $h_{i j}$.

In order to prove uniqueness and well-posedness of the initial value problem, we will need to control the $L^{2}$ size of the solution. This motivates the following definition. 
Definition 2 (Regular Weak Solution) We say a weak solution $\mathbf{u} \in L^{2}\left(0, T ; L^{2}\left(\Sigma, \mathbb{R}^{N}\right)\right)$ is regular if $\mathbf{u}$ satisfies the energy estimate

$$
\|\mathbf{u}\|_{L^{2}\left(0, T ; L^{2}\left(\Sigma, \mathbb{R}^{N}\right)\right)}^{2} \leqslant C\left(\left\|\mathbf{u}_{0}\right\|_{L^{2}\left(\Sigma, \mathbb{R}^{N}\right)}+\int_{0}^{T}\|\mathbf{F}(t, \cdot)\|_{L^{2}\left(\Sigma, \mathbb{R}^{N}\right)}^{2} d t\right)
$$

We may now state our main result concerning solutions of low-regularity symmetric hyperbolic systems.

Theorem 1 Given the linear symmetric hyperbolic system:

$$
\begin{aligned}
L \mathbf{v}=A^{0} \partial_{t} \mathbf{u}+A^{i} \partial_{i} \mathbf{u}+B \mathbf{u} & =\mathbf{F} \\
\mathbf{u}(0, \cdot) & =\mathbf{u}_{0}(\cdot)
\end{aligned}
$$

where $A^{0}, A^{i}, B$ and $\mathbf{F}$ are as above, and the initial data $\mathbf{u}_{0}$ is in $L^{2}\left(\Sigma, \mathbb{R}^{N}\right)$. Then there exists a unique regular weak solution $\mathbf{u} \in L^{2}\left(0, T ; L^{2}\left(\Sigma, \mathbb{R}^{N}\right)\right)$. Furthermore this solution is stable in the sense that the solution depends continuously on the norm of the initial data in $L^{2}\left(\Sigma, \mathbb{R}^{N}\right)$ and the norm of the source function in $L^{2}\left(\Sigma_{[0, T]}, \mathbb{R}^{N}\right)$.

We may now use the above result to establish the following theorem for the wave equation.

Theorem 2 Let $g_{a b}, g^{a b}$ be in $C^{0,1}$, and $f$ in $L^{2}\left(\Sigma_{[0, T]}\right)$. Given initial data $\left(u_{0}, u_{1}\right) \in H^{1}(\Sigma) \times L^{2}(\Sigma)$ then the system

$$
\begin{aligned}
\square_{g} u+m^{2} u & =f \\
u(0, \cdot) & =u_{0} \\
\partial_{t} u(0, \cdot) & =u_{1}
\end{aligned}
$$

has a unique stable solution $u \in H^{1}\left(\Sigma_{[0, T]}\right)$. Moreover, the corresponding energy-momentum tensor $T_{a b}[u]$ is in $L_{l o c}^{\mathrm{f}}\left(\Sigma_{[0, T]}\right)$.

Note that the above result is similar to the one obtained for the homogeneous wave equation in [26]. However, we have extended the results to the more general case that includes mixed space and time derivatives,included a source function and provided a different proof.

We would like to mention that one can not go further without paying a price. As shown by Colombini et al. [27] there are examples of wave equations with coefficients depending only on time of Holder regularity $2 C^{0, \alpha}$ with exponent $\alpha<1$ with no distributional solution. However, they proved well-posedness by moving from Sobolev spaces to Gevrey spaces. These results have been

\footnotetext{
${ }^{2}$ A function $f$ on an open set $\mathcal{U}$ of $\mathbb{R}^{n}$ is said to be Holder or $C^{0, \alpha}$ if there is some non negative constant $K$ such that for each pair of points $p, q \in \mathcal{U},|f(p)-f(q)| \leqslant K|p-q|^{\alpha}$, where $|p|$ denotes the usual Euclidean distance.
} 
further extended to coefficients that depend smoothly in the space variable but are log-Lipschitz (LL) 3 regular in time [28]. In both [27] and [28] the structure of the second order part of the operator considered has the special form

$$
\frac{\partial^{2}}{\partial t^{2}}-\sum_{j, k} \frac{\partial}{\partial x^{j}}\left(a_{j, k}(x, t) \frac{\partial}{\partial x^{k}}\right)
$$

In 29] mixed terms in time and space were allowed and the regularity of the coefficients was LL in space and time. However, the local well posedness results obtained are in Sobolev spaces $H^{s}$ with $|s|<1$ and therefore the energy momentum tensor of the solutions is not integrable. Finally, one can explore wave-type equations with very rough coefficients such as in [30] where one is lead to the necessity of weakening the notion of a solution to the Cauchy problem and enlarging the allowed class of solutions.

\section{Proof of the main theorem}

\subsection{Outline of the proof}

The proof of Theorem 2 uses the vanishing viscosity method described in $\S 7.3$ of Evans [17]. Note however that Evans assumes that the $A^{0}$ and $A^{i}$ have greater regularity than we do and as a result is able to obtain a solution with greater regularity. This explains why our definition of a weak solution has to differ from his. However the essence of the proof is essentially the same. It consists of the following steps:

1. First, we approximate the problem (11) by the system of parabolic initial value-problem on $\Sigma_{[0, T]}$ given by

$$
\begin{aligned}
\partial_{t} \mathbf{u}^{\epsilon}-\epsilon \Delta_{h} \mathbf{u}^{\epsilon}+\left(A^{0}\right)^{-1} A^{i} \partial_{i} \mathbf{u}^{\epsilon}+\left(A^{0}\right)^{-1} B \mathbf{u}^{\epsilon} & =\left(A^{0}\right)^{-1} \mathbf{F} \\
\mathbf{u}^{\epsilon}(0, x) & =\rho^{\epsilon} *\left(\mathbf{u}_{0}(x)\right)
\end{aligned}
$$

where $\left\{\left(\rho^{\epsilon}\right)\right\} \in(0,1]$ is a family of mollifiers. Here $\Delta_{h}$ is the LaplaceBeltrami operator on $\Sigma$ associated with the smooth background Riemannian metric $h_{i j}$. By adding in the second order Laplace-Beltrami terms we obtain a system with smooth principal symbol. We may then use classical methods of parabolic regularity theory to obtain a solution with better analytic properties than the original hyperbolic system.

\footnotetext{
${ }^{3}$ A function $f$ on an open set $\mathcal{U}$ of $\mathbb{R}^{n}$ is said to be log-Lipschitz if there is some non negative constant $K$ such that for each pair of points $p, q \in \mathcal{U},|f(p)-f(q)| \leqslant$ $K|p-q||\ln | p-q||$, where $|p|$ denotes the usual Euclidean distance.
} 
2. Second, we obtain the following uniform energy estimate

$$
\left\|\mathbf{u}^{\epsilon}\right\|_{L^{2}\left(0, T ; L^{2}\left(\Sigma, \mathbb{R}^{N}\right)\right)}^{2} \leqslant C\left(\left\|\mathbf{u}_{0}\right\|_{L^{2}\left(\Sigma, \mathbb{R}^{N}\right)}+\int_{0}^{T}\|\mathbf{F}(t, \cdot)\|_{L^{2}\left(\Sigma, \mathbb{R}^{N}\right)} d t\right) .
$$

where $C$ is independent of $\epsilon$.

3. Third, we take the limit $\epsilon \rightarrow 0$ and show convergence in an appropriate weak sense to a regular weak solution as defined above.

4. Fourth, using the energy inequality (18) we show uniqueness and stability. This concludes the proof of Theorem 1 .

5. Fifth, we rewrite the wave equation as a symmetric hyperbolic problem and show that for a Lipschitz metric the corresponding $L$ satisfies the conditions of Theorem 1.

6. Sixth, we show that the solution of the wave equation obtained via the symmetric hyperbolic problem is in $H^{1}\left(\Sigma_{[0, T]}\right)$. This concludes the proof of Theorem 2 .

\subsection{Approximate solutions and energy estimate}

The results we obtain make extensive use of the vanishing viscosity method. As explained above, the first step is to show that there exist suitable solutions to (17). This step follows directly from the work of Evans ([17, Th. 1 $\S 7.3)$.

Proposition 1 ( Existence of Approximate solutions) For each $\epsilon>0$, there exists a unique solution $\mathbf{u}^{\epsilon}$ of (17) with $\mathbf{u}^{\epsilon} \in L^{2}\left(0, T ; H^{2}\left(\Sigma, \mathbb{R}^{N}\right)\right)$ and $\dot{\mathbf{u}}^{\epsilon} \in L^{2}\left(0, T ; L^{2}\left(\Sigma, \mathbb{R}^{N}\right)\right)$.

Proof. This is a variant of a standard result for parabolic systems. Following Evans set $X=L^{\infty}\left(0, T ; H^{1}\left(\Sigma, \mathbb{R}^{N}\right)\right)$ and then for each $\mathbf{w} \in X$, consider the linear system

$$
\begin{aligned}
\partial_{t} \mathbf{u}^{\epsilon}-\epsilon \Delta_{h} \mathbf{u}^{\epsilon} & =-\left(A^{0}\right)^{-1} A^{i} \partial_{i} \mathbf{w}-\left(A^{0}\right)^{-1} B \mathbf{w}+\left(A^{0}\right)^{-1} \mathbf{F} \\
\mathbf{u}^{\epsilon}(0, x) & =\mathbf{u}_{0}^{\epsilon}(x)
\end{aligned}
$$

where $\mathbf{u}_{0}^{\epsilon}(x)=\rho^{\epsilon}(x) *\left(\mathbf{u}_{0}(x)\right)$. Notice that the system is formed of $N$ scalar parabolic equations of the form $\partial_{t} v-\epsilon \Delta_{h} v=f$. The coefficients are now all smooth and the only loss of regularity comes from the source term on the RHS of (19). However as this is bounded in $L^{2}\left(0, T ; L^{2}\left(\Sigma, \mathbb{R}^{N}\right)\right)$, we can apply standard results (see e.g. [17] Th. 5 §7.1) to show that there is a unique solution $\mathbf{u}^{\epsilon} \in L^{2}\left(0, T ; H^{2}\left(\Sigma, \mathbb{R}^{N}\right)\right)$ with $\dot{\mathbf{u}}^{\epsilon} \in L^{2}\left(0, T ; L^{2}\left(\Sigma, \mathbb{R}^{N}\right)\right)$.

In the same manner we can choose $\tilde{\mathbf{w}} \in X$ and find $\tilde{\mathbf{u}}^{\epsilon}$ that solves 


$$
\begin{aligned}
\partial_{t} \tilde{\mathbf{u}}^{\epsilon}-\epsilon \Delta_{h} \tilde{\mathbf{u}}^{\epsilon} & =-\left(A^{0}\right)^{-1} A^{i} \partial_{i} \tilde{\mathbf{w}}-\left(A^{0}\right)^{-1} B \tilde{\mathbf{w}}+\left(A^{0}\right)^{-1} \mathbf{F} \\
\tilde{\mathbf{u}}^{\epsilon}(0, x) & =\mathbf{u}_{0}^{\epsilon}(x)
\end{aligned}
$$

Subtracting $\mathbf{u}^{\epsilon}-\tilde{\mathbf{u}}^{\epsilon}$, we find $\overline{\mathbf{u}}^{\epsilon}=\mathbf{u}^{\epsilon}-\tilde{\mathbf{u}}^{\epsilon}$ solves

$$
\begin{aligned}
\partial_{t} \overline{\mathbf{u}}^{\epsilon}-\epsilon \Delta_{h} \overline{\mathbf{u}}^{\epsilon} & =-\left(A^{0}\right)^{-1} A^{i} \partial_{i} \overline{\mathbf{w}}-\left(A^{0}\right)^{-1} B \overline{\mathbf{w}} \\
\overline{\mathbf{u}}^{\epsilon}(0, x) & =0
\end{aligned}
$$

where $\overline{\mathbf{w}}=\mathbf{w}-\tilde{\mathbf{w}}$. Using standard energy estimates for solutions of parabolic equations we have that $\overline{\mathbf{u}}$ satisfies:

$$
\begin{gathered}
\sup _{0 \leqslant t \leqslant T}\left\|\overline{\mathbf{u}}^{\epsilon}(t)\right\|_{H^{1}\left(\Sigma, \mathbb{R}^{N}\right)}^{2} \\
\leqslant C(T, \epsilon)\left(\left\|\left(A^{0}\right)^{-1}\left(A^{i} \partial_{i} \overline{\mathbf{w}}+B \overline{\mathbf{w}}\right)\right\|_{L^{2}\left(0, T ; L^{2}\left(\Sigma, \mathbb{R}^{N}\right)\right)}^{2}\right) \\
\leqslant C(T, \epsilon)\left(\sup _{0 \leqslant t \leqslant T}\|\overline{\mathbf{w}}(t)\|_{H^{1}\left(\Sigma, \mathbb{R}^{N}\right)}^{2}\right)
\end{gathered}
$$

Thus

$$
\left\|\overline{\mathbf{u}}^{\epsilon}\right\|_{L^{\infty}\left(0, T ;\left(\Sigma, \mathbb{R}^{N}\right)\right)} \leqslant C(T, \epsilon)\|\overline{\mathbf{w}}\|_{L^{\infty}\left(0, T ;\left(\Sigma, \mathbb{R}^{N}\right)\right)}
$$

Therefore, if $T$ is small enough such that $C(T, \epsilon) \leqslant \frac{1}{2}$ we obtain that

$$
\left\|\overline{\mathbf{u}}^{\epsilon}\right\|_{L^{\infty}\left(0, T ;\left(\Sigma, \mathbb{R}^{N}\right)\right)} \leqslant \frac{1}{2}\|\overline{\mathbf{w}}\|_{L^{\infty}\left(0, T ;\left(\Sigma, \mathbb{R}^{N}\right)\right)}
$$

so that

$$
\left\|\mathbf{u}^{\epsilon}-\tilde{\mathbf{u}}^{\epsilon}\right\|_{L^{\infty}\left(0, T ;\left(\Sigma, \mathbb{R}^{N}\right)\right)} \leqslant \frac{1}{2}\|\mathbf{w}-\tilde{\mathbf{w}}\|_{L^{\infty}\left(0, T ;\left(\Sigma, \mathbb{R}^{N}\right)\right)}
$$

This implies that we have a contraction mapping and the hypothesis of Banach's fixed point theorem is satisfied for the mapping

$$
\begin{aligned}
M: L^{\infty}\left(0, T ;\left(\Sigma, \mathbb{R}^{N}\right)\right) & \rightarrow L^{\infty}\left(0, T ;\left(\Sigma, \mathbb{R}^{N}\right)\right) \\
\mathbf{w} & \mapsto \mathbf{u}^{\epsilon}
\end{aligned}
$$

which therefore has a unique fixed point which solves (17). If $C(T, \epsilon)>\frac{1}{2}$ we can choose $T_{1}$ small enough such that $C\left(T_{1}, \epsilon\right) \leqslant \frac{1}{2}$ and then repeat the above argument for intervals $\left[0, T_{1}\right],\left[T_{1}, 2 T_{1}\right], \ldots,\left[n T_{1}, T\right]$. In either case we obtain a solution $\mathbf{u}^{\epsilon}$ which solves (17) on the interval $[0, T]$. Standard parabolic regularity theory (see e.g. Th. $5 \$ 7.1$ [17]) then gives us $\mathbf{u}^{\epsilon} \in$ $L^{2}\left(0, T ; H^{2}\left(\Sigma, \mathbb{R}^{N}\right)\right)$ and that $\dot{\mathbf{u}}^{\epsilon} \in L^{2}\left(0, T ; L^{2}\left(\Sigma, \mathbb{R}^{N}\right)\right)$, which concludes the proof of Theorem $1 \square$

Note that Evans goes on to use Th. $6 \$ 7.1$ 17 to obtain an improved regularity result showing that $\mathbf{u}^{\epsilon} \in L^{2}\left(0, T ; H^{3}\left(\Sigma, \mathbb{R}^{N}\right)\right)$ and that the time derivative $\dot{\mathbf{u}}^{\epsilon} \in L^{2}\left(0, T ; H^{1}\left(\Sigma, \mathbb{R}^{N}\right)\right)$. However we do not need this result. 


\subsection{Energy estimates}

The next step is to obtain the uniform energy estimate in $\epsilon$ for the solutions $\mathbf{u}^{\epsilon}$. This is the content of the following proposition.

Proposition 2 There exists a constant $C$ depending on $T, \Sigma, h_{i j}, \partial_{l} h_{i j}$, $\sup _{x \in \Sigma_{[0, T]}}\left(\left|\left(A^{0}\right)^{-1}\right|,\left|A^{i}\right|,|B|,\left|\partial_{t}\left(A^{0}\right)^{-1}\right|,\left|\partial_{t} A^{i}\right|,\left|\partial_{j}\left(A^{0}\right)^{-1}\right|,\left|\partial_{j} A^{i}\right|\right)$ such that

$$
\left\|\mathbf{u}^{\epsilon}\right\|_{L^{2}\left(0, T ; L^{2}\left(\Sigma, \mathbb{R}^{N}\right)\right)}^{2} \leqslant C\left(\left\|\mathbf{u}_{0}\right\|_{L^{2}\left(\Sigma, \mathbb{R}^{N}\right)}+\int_{0}^{T}\|\mathbf{F}(t, \cdot)\|_{L^{2}\left(\Sigma, \mathbb{R}^{N}\right)} d t\right)
$$

Therefore the estimate is independent of $\epsilon$.

Proof of Proposition 囵. Taking the time derivative of $\| \mathbf{u}^{\epsilon}||_{L^{2}\left(\Sigma, \mathbb{R}^{N}\right)}^{2}$ gives:

$$
\begin{aligned}
& \frac{d}{d t}\left(\left\|\mathbf{u}^{\epsilon}(t)\right\|_{L^{2}\left(\Sigma, \mathbb{R}^{N}\right)}^{2}\right)=2\left(\mathbf{u}^{\epsilon}, \dot{\mathbf{u}}^{\epsilon}\right)_{L^{2}\left(\Sigma, \mathbb{R}^{N}\right)} \\
& =2\left(\mathbf{u}^{\epsilon}, \epsilon \Delta_{h} \mathbf{u}^{\epsilon}-\left(A^{0}\right)^{-1} A^{i} \partial_{i} \mathbf{u}^{\epsilon}-\left(A^{0}\right)^{-1} B \mathbf{u}^{\epsilon}+\left(A^{0}\right)^{-1} \mathbf{F}\right)_{L^{2}\left(\Sigma, \mathbb{R}^{N}\right)}
\end{aligned}
$$

We have estimates for the following terms in (30)

$$
\begin{aligned}
\left|\left(\mathbf{u}^{\epsilon},\left(A^{0}\right)^{-1} \mathbf{F}\right)_{L^{2}\left(\Sigma, \mathbb{R}^{N}\right)}\right| & \leqslant C_{1}\left(\left\|\mathbf{u}^{\epsilon}(t)\right\|_{L^{2}\left(\Sigma, \mathbb{R}^{N}\right)}^{2}+\|\mathbf{F}\|_{L^{2}\left(\Sigma, \mathbb{R}^{N}\right)}^{2}\right) \\
\left(\mathbf{u}^{\epsilon}, \epsilon \Delta_{h} \mathbf{u}^{\epsilon}\right)_{L^{2}\left(\Sigma, \mathbb{R}^{N}\right)} & =-\epsilon \sum_{l=1}^{N} \int_{\Sigma} h^{i j} \partial_{i}\left(u^{l}\right) \partial_{j}\left(u^{l}\right) \nu_{h} \leqslant 0 \\
\left|\left(\mathbf{u}^{\epsilon},\left(A^{0}\right)^{-1} B \mathbf{u}^{\epsilon}\right)\right| & \leqslant C_{2}\left\|\mathbf{u}^{\epsilon}\right\|_{L^{2}\left(\Sigma, \mathbb{R}^{N}\right)}^{2} .
\end{aligned}
$$

However we also require an estimate for $\left(\mathbf{u}^{\epsilon},\left(A^{0}\right)^{-1} A^{i} \partial_{i} \mathbf{u}^{\epsilon}\right)_{L^{2}\left(\Sigma, \mathbb{R}^{N}\right)}$, which is the remaining term in (30). This term can be estimated by applying a suitable integration by parts.

We first assume that the smooth background Riemannian metric is $h_{i j}=\delta_{i j}$, write $\left(A^{0}\right)^{-1} A^{i}$ as $\tilde{A}^{i}$ and estimate $\left(\mathbf{u}^{\epsilon}, \tilde{A}^{i} \partial_{i} \mathbf{u}^{\epsilon}\right)_{L^{2}\left(\Sigma, \mathbb{R}^{N}\right)}$. Using the fact that the $\tilde{A}^{i}$ are symmetric, we then have

$$
\begin{array}{cc} 
& \left(\mathbf{u}^{\epsilon}, \tilde{A}^{i} \partial_{i} \mathbf{u}^{\epsilon}\right)_{L^{2}\left(\Sigma, \mathbb{R}^{N}\right)} \\
= & \int_{\Sigma} \mathbf{u}^{\epsilon} \cdot\left(\tilde{A}^{i} \partial_{i} \mathbf{u}^{\epsilon}\right) d^{n} x \\
= & \frac{1}{2} \int_{\Sigma} \partial_{i}\left(\tilde{A}^{i} \mathbf{u}^{\epsilon}\right) \cdot \mathbf{u}^{\epsilon} d^{3} x-\frac{1}{2} \int_{\Sigma}\left(\partial_{i} \tilde{A}^{i}\right) \mathbf{u}^{\epsilon} \cdot \mathbf{u}^{\epsilon} d^{n} x \\
= & -\frac{1}{2} \int_{\Sigma}\left(\partial_{i} \tilde{A}^{i}\right) \mathbf{u}^{\epsilon} \cdot \mathbf{u}^{\epsilon} d^{3} x
\end{array}
$$

So that

$$
\begin{aligned}
\left|\left(\mathbf{u}^{\epsilon}, \tilde{A}^{i} \partial_{i} \mathbf{u}^{\epsilon}\right)_{L^{2}\left(\Sigma, \mathbb{R}^{N}\right)}\right| & \leqslant \frac{1}{2}\left|\int_{\Sigma}\left(\partial_{i} \tilde{A}^{i}\right) \mathbf{u}^{\epsilon} \cdot \mathbf{u}^{\epsilon} d^{n} x\right| \\
& \leqslant C_{3}\left\|\mathbf{u}^{\epsilon}\right\|_{L^{2}\left(\Sigma, \mathbb{R}^{N}\right)}^{2}
\end{aligned}
$$


where the constant $C_{3}$ is independent of $\epsilon$ and we have used the fact that $\partial_{i} \tilde{A}^{i}$ is bounded. In the case of a general background Riemannian metric $h_{i j}$ rather than the ordinary divergence of $\tilde{A}^{i}$, one obtains the divergence with respect to the background metric $h_{i j}$ and the corresponding result is

$$
\begin{aligned}
\left|\left(\mathbf{v}, \tilde{A}^{i} \partial_{i} \mathbf{v}\right)_{L^{2}\left(\Sigma, \mathbb{R}^{N}\right)}\right| & \leqslant \frac{1}{2}\left|\int_{\Sigma}\left(\left(\partial_{i}+\tilde{\Gamma}_{k i}^{k}\right) \tilde{A}^{i}\right) \mathbf{u}^{\epsilon} \cdot \mathbf{u}^{\epsilon} \nu_{h}\right| \\
& \leqslant C_{4}|| \mathbf{u}^{\epsilon} \|_{L^{2}\left(\Sigma, \mathbb{R}^{N}\right)}^{2}
\end{aligned}
$$

where again $C_{4}$ is independent of $\epsilon$. For the case where $\Sigma$ is an open, bounded set of $\mathbb{R}^{n}$ with smooth boundary $\partial \Sigma$, one needs a slightly more complicated argument where one approximates $\mathbf{u}^{\epsilon}$ by smooth functions of compact support (see [17] $\$ 7.3$ for details).

Using all the available estimates (31), (32), (33) and (40) in (30) we obtain the estimate

$$
\frac{d}{d t}\left(\left\|\mathbf{u}^{\epsilon}(t)\right\|_{L^{2}\left(\Sigma, \mathbb{R}^{N}\right)}^{2}\right) \leqslant C_{5}\left(\left\|\mathbf{u}^{\epsilon}\right\|_{L^{2}\left(\Sigma, \mathbb{R}^{N}\right)}^{2}+\|\mathbf{F}\|_{L^{2}\left(\Sigma, \mathbb{R}^{N}\right)}^{2}\right)
$$

Using Gronwalls's inequality and the fact that

$$
\left\|\mathbf{u}^{\epsilon}(0, x)\right\|_{L^{2}\left(\Sigma, \mathbb{R}^{N}\right)} \leqslant\left\|\mathbf{u}_{0}(x)\right\|_{L^{2}\left(\Sigma, \mathbb{R}^{N}\right)}
$$

we obtain the estimate

$$
\sup _{0 \leqslant t \leqslant T}\left\|\mathbf{u}^{\epsilon}(t)\right\|_{L^{2}\left(\Sigma, \mathbb{R}^{N}\right)}^{2} \leqslant C_{6}\left(\left\|\mathbf{u}_{0}\right\|_{L^{2}\left(\Sigma, \mathbb{R}^{N}\right)}^{2}+\int_{0}^{T}\|\mathbf{F}\|_{L^{2}\left(\Sigma, \mathbb{R}^{N}\right)}^{2} d t\right)
$$

Finally noting that

$$
\left\|\mathbf{u}^{\epsilon}\right\|_{L^{2}\left(0, T ; L^{2}\left(\Sigma, \mathbb{R}^{N}\right)\right)}^{2} \leqslant T \sup _{0 \leqslant t \leqslant T}\left\|\mathbf{u}^{\epsilon}(t)\right\|_{L^{2}\left(\Sigma, \mathbb{R}^{N}\right)}^{2}
$$

and using this in the estimate (44) we obtain (29) which concludes the proof.

\subsection{Existence, Uniqueness and Stability}

In this section we show the existence and uniqueness of the initial value problem. In Proposition 1 we obtained solutions $\mathbf{u}^{\epsilon}$ in $L^{2}\left(0, T ; H^{2}\left(\Sigma, \mathbb{R}^{N}\right)\right)$ to the parabolic system (17). Using the Banach-Alaoglu Theorem there exists a subsequence $\left\{\mathbf{u}^{\epsilon_{k}}\right\}_{k=1}^{\infty}$ that converges weakly to a function $\mathbf{u}$ in $L^{2}\left(0, T ; L^{2}\left(\Sigma, \mathbb{R}^{\mathbb{N}}\right)\right)$. We now show that this converges to a weak solution of (11).

First we choose a function $\tilde{\mathbf{w}} \in \tilde{H}^{2}\left(\Sigma_{[0, T]}, \mathbb{R}^{N}\right)$, take the dot product with equation (17) and integrate over $t$ and $x$. This gives 


$$
\begin{aligned}
& \int_{0}^{T}\left(\left(A^{0}\right)^{-1} L \mathbf{u}^{\epsilon}, \tilde{\mathbf{w}}\right)_{L^{2}\left(\Sigma, \mathbb{R}^{N}\right)}-\epsilon\left(\Delta_{h} \mathbf{u}^{\epsilon}, \tilde{\mathbf{w}}\right)_{L^{2}\left(\Sigma, \mathbb{R}^{N}\right)} d t \\
= & \int_{0}^{T}\left(\left(A^{0}\right)^{-1} \mathbf{F}, \tilde{\mathbf{w}}\right)_{L^{2}\left(\Sigma, \mathbb{R}^{N}\right)} d t
\end{aligned}
$$

Integrating by parts we obtain

$$
\begin{aligned}
& \int_{0}^{T}\left(\mathbf{u}^{\epsilon}, \tilde{L} \tilde{\mathbf{w}}\right)_{L^{2}\left(\Sigma, \mathbb{R}^{N}\right)}-\epsilon\left(\mathbf{u}^{\epsilon}, \Delta_{h} \tilde{\mathbf{w}}\right)_{L^{2}\left(\Sigma, \mathbb{R}^{N}\right)} d t \\
= & \int_{0}^{T}\left(\left(A^{0}\right)^{-1} \mathbf{F}, \tilde{\mathbf{w}}\right)_{L^{2}\left(\Sigma, \mathbb{R}^{N}\right)} d t+\left(\mathbf{u}^{\epsilon}(0), \tilde{\mathbf{w}}(0)\right)_{L^{2}\left(\Sigma, \mathbb{R}^{N}\right)}
\end{aligned}
$$

where the operator $\tilde{L}$ is defined by

$$
\tilde{L} \tilde{\mathbf{w}}:=-\partial_{t} \tilde{\mathbf{w}}-\partial_{i}\left(A^{i}\left(A^{0}\right)^{-1} \tilde{\mathbf{w}}\right)+B^{T}\left(\left(A^{0}\right)^{-1}\right) \tilde{\mathbf{w}}-\tilde{\Gamma}_{i l}^{l} A^{i}\left(A^{0}\right)^{-1} \tilde{\mathbf{w}}
$$

Then taking the limit $k \rightarrow \infty$ and using the weak convergence of $\mathbf{u}^{\epsilon_{k}} \rightarrow \mathbf{u}$ and that $\mathbf{u}^{\epsilon_{k}}(0) \rightarrow\left(A^{0}\right)^{-1}(0) \mathbf{u}_{0}$ in $L^{2}\left(\Sigma, \mathbb{R}^{N}\right)$ we obtain

$$
\begin{aligned}
& \int_{0}^{T}(\mathbf{u}, \tilde{L} \tilde{\mathbf{w}})_{L^{2}\left(\Sigma, \mathbb{R}^{N}\right)} d t \\
= & \int_{0}^{T}\left(\left(A^{0}\right)^{-1} \mathbf{F}, \tilde{\mathbf{w}}\right)_{L^{2}\left(\Sigma, \mathbb{R}^{N}\right)} d t+\left(\mathbf{u}_{0}, \tilde{\mathbf{w}}(0)\right)_{L^{2}\left(\Sigma, \mathbb{R}^{N}\right)}
\end{aligned}
$$

The above equality was obtained for $\tilde{\mathbf{w}} \in \tilde{H}^{2}\left(\Sigma_{[0, T]}, \mathbb{R}^{N}\right)$, however the equation remains well-defined for $\tilde{\mathbf{w}} \in \tilde{H}^{1}\left(\Sigma_{[0, T]}, \mathbb{R}^{N}\right) \subset C^{0}\left([0, T], L^{2}(\Sigma)\right)$. We now show that not only is the equation well-defined, but it remains valid for $\tilde{\mathbf{w}} \in \tilde{H}^{1}\left(\Sigma_{[0, T]}, \mathbb{R}^{N}\right)$. The method involves taking the convolution with a family of mollifiers $\rho^{\delta}$ and then passing to the limit $\delta \rightarrow 0$.

Let $\tilde{\mathbf{w}} \in \tilde{H}^{1}\left(\Sigma_{[0, T]}, \mathbb{R}^{N}\right)$ and define $\tilde{\mathbf{w}}^{\delta}=\rho^{\delta} * \tilde{\mathbf{w}}$ where we have chosen $\delta$ close enough to zero such that $\tilde{\mathbf{w}}^{\delta}(T, \cdot)=0$. Therefore we have

$$
\begin{aligned}
& \int_{0}^{T}\left(\mathbf{u}, \tilde{L} \tilde{\mathbf{w}}^{\delta}\right)_{L^{2}\left(\Sigma, \mathbb{R}^{N}\right)} d t \\
= & \int_{0}^{T}\left(\left(A^{0}\right)^{-1} \mathbf{F}, \tilde{\mathbf{w}}^{\delta}\right)_{L^{2}\left(\Sigma, \mathbb{R}^{N}\right)} d t+\left(\mathbf{u}(0), \tilde{\mathbf{w}}^{\delta}(0)\right)_{L^{2}\left(\Sigma, \mathbb{R}^{N}\right)}
\end{aligned}
$$

Taking the limit $\delta \rightarrow 0$ and using the Schwartz inequality we have the following limits

$$
\begin{aligned}
\tilde{\mathbf{w}}_{t}^{\delta} & \rightarrow \tilde{\mathbf{w}}_{t} \text { in } L^{2}\left(0, T ; L^{2}\left(\Sigma, \mathbb{R}^{N}\right)\right) \\
-\left(A^{i}\left(A^{0}\right)^{-1}\right) \tilde{\mathbf{w}}_{i}^{\delta} & \rightarrow-\left(A^{i}\left(A^{0}\right)^{-1}\right) \tilde{\mathbf{w}}_{i} \text { in } L^{2}\left(0, T ; L^{2}\left(\Sigma, \mathbb{R}^{N}\right)\right) \\
B^{T}\left(\left(A^{0}\right)^{-1}\right) \tilde{\mathbf{w}}^{\delta} & \rightarrow B^{T}\left(\left(A^{0}\right)^{-1}\right) \tilde{\mathbf{w}} \text { in } L^{2}\left(0, T ; L^{2}\left(\Sigma, \mathbb{R}^{N}\right)\right) \\
\tilde{\Gamma}_{l k}^{k} A^{l}\left(A^{0}\right)^{-1} \tilde{\mathbf{w}}^{\delta} & \rightarrow \tilde{\Gamma}_{l k}^{k} A^{l}\left(A^{0}\right)^{-1} \tilde{\mathbf{w}} \text { in } L^{2}\left(0, T ; L^{2}\left(\Sigma, \mathbb{R}^{N}\right)\right) \\
\tilde{\mathbf{w}}^{\delta}(0) & \rightarrow \tilde{\mathbf{w}}(0) \text { in } L^{2}\left(\Sigma, \mathbb{R}^{N}\right)
\end{aligned}
$$


We therefore conclude that

$$
\begin{aligned}
& \int_{0}^{T}(\mathbf{u}, \tilde{L} \tilde{\mathbf{w}})_{L^{2}\left(\Sigma, \mathbb{R}^{N}\right)} d t \\
= & \int_{0}^{T}\left(\left(A^{0}\right)^{-1} \mathbf{F}, \tilde{\mathbf{w}}\right)_{L^{2}\left(\Sigma, \mathbb{R}^{N}\right)} d t+\left(\mathbf{u}_{0}, \tilde{\mathbf{w}}(0)\right)_{L^{2}\left(\Sigma, \mathbb{R}^{N}\right)}
\end{aligned}
$$

for $\tilde{\mathbf{w}} \in \tilde{H}^{1}\left(\Sigma_{[0, T]}, \mathbb{R}^{N}\right)$

If we now take $\mathbf{w} \in C^{\infty}\left(\Sigma_{[0, T]}, \mathbb{R}^{N}\right)$, with $\operatorname{supp}(\mathbf{w}) \subseteq[0, T) \times \Sigma$, and multiply it by $A^{0}$ we obtain that $A^{0} \mathbf{w} \in \tilde{W}^{1, \infty}\left(\Sigma_{[0, T]}, \mathbb{R}^{N}\right) \subset \tilde{H}^{1}\left(\Sigma_{[0, T]}, \mathbb{R}^{N}\right)$ and $\operatorname{supp}\left(A^{0} \mathbf{w}\right) \subset \operatorname{supp}(\mathbf{w})$.

We may therefore insert $\tilde{\mathbf{w}}=A^{0} \mathbf{w}$ in (56) which gives

$$
\begin{aligned}
& \int_{0}^{T}\left(\mathbf{u}, \tilde{L} A^{0} \mathbf{w}\right)_{L^{2}\left(\Sigma, \mathbb{R}^{N}\right)} d t \\
= & \int_{0}^{T}\left(\left(A^{0}\right)^{-1} \mathbf{F},\left(A^{0}\right) \mathbf{w}\right)_{L^{2}\left(\Sigma, \mathbb{R}^{N}\right)} d t+\left(\mathbf{u}_{0},\left.\left(A^{0}\right) \mathbf{w}\right|_{t=0}\right)_{L^{2}\left(\Sigma, \mathbb{R}^{N}\right)}
\end{aligned}
$$

which can be rewritten as

$$
\int_{0}^{T}\left(\mathbf{u}, L^{*} \mathbf{w}\right)_{L^{2}\left(\Sigma, \mathbb{R}^{N}\right)} d t=\int_{0}^{T}(\mathbf{F}, \mathbf{w})_{L^{2}\left(\Sigma, \mathbb{R}^{N}\right)} d t+\left(\left(A^{0}(0)\right) \mathbf{u}_{0}, \mathbf{w}(0)\right)_{L^{2}\left(\Sigma, \mathbb{R}^{N}\right)}
$$

for all $\mathbf{w} \in C^{\infty}\left(\Sigma_{[0, T]}, \mathbb{R}^{N}\right)$ with $\operatorname{supp}(\mathbf{w}) \subseteq[0, T) \times \Sigma$, where $L^{*}$ is the formal adjoint defined by equation (9).

We have therefore proved that the $\mathbf{u}$ obtained by taking the limit of the subsequence $\left\{\mathbf{u}^{\epsilon_{k}}\right\}_{k=1}^{\infty}$ is a weak solution lying in $L^{2}\left(0, T ; L^{2}\left(\Sigma, \mathbb{R}^{\mathbb{N}}\right)\right)$ with initial data $\mathbf{u}_{0}$ as in Definition 1.

As the norm function is lower semi continuous, we may take the limit of equation (29) to obtain the estimate

$$
\begin{aligned}
\|\mathbf{u}\|_{L^{2}\left(0, T ; L^{2}\left(\Sigma, \mathbb{R}^{N}\right)\right)}^{2} & \leqslant \lim _{k \rightarrow \infty}\left\|\mathbf{u}^{\epsilon_{k}}\right\|_{L^{2}\left(0, T ; L^{2}\left(\Sigma, \mathbb{R}^{N}\right)\right)}^{2} \\
& \leqslant C\left(\left\|\mathbf{u}_{0}\right\|_{L^{2}\left(\Sigma, \mathbb{R}^{N}\right)}^{2}+\int_{0}^{T}\|\mathbf{F}\|_{L^{2}\left(\Sigma, \mathbb{R}^{N}\right)}^{2} d t\right)
\end{aligned}
$$

So that the solution we have obtained is a regular weak solution.

To show uniqueness we consider two functions $\mathbf{u}_{1}, \mathbf{u}_{2}$ which are both regular weak solutions. Then $\mathbf{u}=\mathbf{u}_{1}-\mathbf{u}_{2}$ is a regular weak solution with source 
$\mathbf{F}=0$ and initial data $\mathbf{u}_{0}=0$. Moreover the solution satisfies the energy estimate (10) as shown above. Therefore

$$
\|\left.\mathbf{u}\right|_{L^{2}\left(0, T ; L^{2}\left(\Sigma, \mathbb{R}^{N}\right)\right)}=0
$$

This implies $\mathbf{u}=0$ and therefore $\mathbf{u}_{1}=\mathbf{u}_{2}$.

The final step in establishing well-posedness is to prove the stability of the solution with respect to initial data. To make the concept precise we say that the solution $\mathbf{u}$ is continuously stable in $L^{2}\left(0, T ; L^{2}\left(\Sigma, \mathbb{R}^{N}\right)\right)$ with respect to initial data $\mathbf{u}_{0}$ in $L^{2}\left(\Sigma, \mathbb{R}^{N}\right)$, if given $\epsilon>0$ there is a $\delta$ depending on $\mathbf{u}_{0}$ such that if $\left.\tilde{\mathbf{u}}_{0} \in L^{2}\left(\Sigma, \mathbb{R}^{N}\right)\right)$ with:

$$
\left\|\tilde{\mathbf{u}}_{0}-\mathbf{u}_{0}\right\|_{L^{2}\left(\Sigma, \mathbb{R}^{N}\right)} \leqslant \delta,
$$

then the corresponding weak solution $\tilde{\mathbf{u}}$ with source function $\mathbf{F}$ satisfies

$$
\|\tilde{\mathbf{u}}-\mathbf{u}\|_{L^{2}\left(0, T ; L^{2}\left(\Sigma, \mathbb{R}^{N}\right)\right)} \leqslant \epsilon
$$

The stability results follows from using the energy inequality shown in Theorem 2 for the difference $\tilde{\mathbf{u}}-\mathbf{u}$ which gives

$$
\|\tilde{\mathbf{u}}-\mathbf{u}\|_{L^{2}\left(0, T ; L^{2}\left(\Sigma, \mathbb{R}^{N}\right)\right)}^{2} \leqslant C\left\|\tilde{\mathbf{u}_{0}}-\mathbf{u}_{0}\right\|_{L^{2}\left(\Sigma, \mathbb{R}^{N}\right)}^{2}
$$

Now choosing $\delta=\frac{\epsilon}{C}$ we obtain the inequality:

$$
\|\tilde{\mathbf{u}}-\mathbf{u}\|_{L^{2}\left(0, T ; L^{2}\left(\Sigma, \mathbb{R}^{N}\right)\right)}^{2} \leqslant \epsilon^{2}
$$

which establishes stability with respect to the initial data.

This concludes the proof of Theorem 1 .

\subsection{The wave equation}

In order to apply the results of Theorem 1 to applications in general relativity we will show here how the wave equation can be written as a first order linear symmetric problem.

We define

$$
\mathbf{v}=\left(\partial_{1} u, \ldots, \partial_{n} u, \partial_{t} u, u\right)^{T}=\left(v^{1}, \ldots, v^{n}, v^{n+1}, v^{n+2}\right)^{T} \in \mathbb{R}^{n+2}
$$

and the symmetric $(n+2) \times(n+2)$ matrices $A^{\mu}$ by:

$$
A^{0}=\left(\begin{array}{ccccc}
g^{11} & g^{12} & \ldots & 0 & 0 \\
g^{21} & g^{22} & \ldots & 0 & 0 \\
\vdots & \vdots & \ddots & \vdots & \vdots \\
0 & 0 & \cdots & -g^{00} & 0 \\
0 & 0 & \cdots & 0 & 1
\end{array}\right)
$$


$A^{k}=\left(\begin{array}{ccccc}0 & 0 & \cdots & g^{1 k} & 0 \\ 0 & 0 & \cdots & g^{2 k} & 0 \\ \vdots & \vdots & \ddots & \vdots & \vdots \\ g^{1 k} & g^{2 k} & \cdots & 2 g^{0 k} & 0 \\ 0 & 0 & \cdots & 0 & 0\end{array}\right)$

We further define the matrix $B$ to be given by

$B=\left(\begin{array}{ccccc}0 & 0 & \cdots & 0 & 0 \\ 0 & 0 & \cdots & 0 & 0 \\ \vdots & \vdots & \ddots & \vdots & 0 \\ g^{a b} \Gamma_{a b}^{1} & g^{a b} \Gamma_{a b}^{2} & \cdots & g^{a b} \Gamma_{a b}^{0} & -m^{2} \\ 0 & 0 & \cdots & -1 & 0\end{array}\right)$

where $\Gamma_{a b}^{c}$ are the connection coefficients of the spacetime metric $g_{a b}$. We also define the $\mathbb{R}^{N}$-valued vector function $\mathbf{F}$ by $\mathbf{F}=(0,0, \ldots,-f, 0)^{T}$.

In this way, we may rewrite the scalar wave equation (13) as a first order system which has the form

$$
\begin{aligned}
L \mathbf{v}=A^{0} \partial_{t} \mathbf{v}-A^{i} \partial_{i} \mathbf{v}+B \mathbf{v} & =\mathbf{F} \\
\mathbf{v}(0, \cdot) & =\mathbf{v}_{0}(\cdot)
\end{aligned}
$$

We may then use the Theorem 1 to establish well-posedness of (67). To prove existence of solutions to the wave equation, we therefore need to prove that the solution $\mathbf{v}$ of the symmetric hyperbolic system (67) has the form $\mathbf{v}=\left(\partial_{1} u, \ldots, \partial_{n} u, \partial_{t} u, u\right)^{T}$.

We now mollify our solution $\mathbf{v}$ using a strict delta net to obtain a sequence of smooth functions $\mathbf{v}^{\epsilon}=\rho^{\epsilon} * \mathbf{v}=\left(v_{\epsilon}^{1}, \ldots, v_{\epsilon}^{n+2}\right)^{T}$ that satisfies:

$$
\int_{0}^{T}\left(L \mathbf{v}^{\epsilon}, \mathbf{w}\right)_{L^{2}\left(\Sigma, \mathbb{R}^{N}\right)} d t=\int_{0}^{T}\left(\mathbf{F}^{\epsilon}, \mathbf{w}\right)_{L^{2}\left(\Sigma, \mathbb{R}^{N}\right)} d t
$$

for a suitable mollified $\mathbf{F}^{\epsilon}$ of the form $\left(0,0, \ldots,-h^{\epsilon}, 0\right)^{T}$ where $h^{\epsilon}$ is any $L^{2}$ function.

Then

$$
\int_{0}^{T}\left(L \mathbf{v}^{\epsilon}-\mathbf{F}^{\epsilon}, \mathbf{w}\right)_{L^{2}\left(\Sigma, \mathbb{R}^{N}\right)} d t=0
$$

for all $\mathbf{w} \in C^{\infty}\left(\Sigma_{[0, T]}, \mathbb{R}^{N}\right)$ and therefore $L \mathbf{v}^{\epsilon}=\mathbf{F}^{\epsilon}$ almost everywhere so we obtain that:

$$
\partial_{t} v_{\epsilon}^{j}=\partial_{j} v_{\epsilon}^{n+1}
$$

and

$$
\partial_{t} v_{\epsilon}^{n+2}=v_{\epsilon}^{n+1}
$$


Also, we choose initial data such that

$$
\partial_{i} v_{\epsilon}^{n+2}(0, \cdot)=v_{\epsilon}^{i}(0, \cdot), \quad i=1,2, \ldots, n
$$

We now define $u_{\epsilon}=v_{\epsilon}^{n+2}=\rho^{\epsilon} * v^{n+2}$ and obtain

$$
\begin{aligned}
\partial_{i} u_{\epsilon}(\tau) & =v_{\epsilon}^{i}(0, \cdot)+\int_{0}^{\tau} \partial_{i} \partial_{t} v_{\epsilon}^{n+2} d t \\
& =v_{\epsilon}^{i}(0, \cdot)+\int_{0}^{\tau} \partial_{t} v_{\epsilon}^{i} d t \\
& =v_{\epsilon}^{i}(\tau)
\end{aligned}
$$

Taking now into account that $\mathbf{v}^{\epsilon} \rightarrow \mathbf{v}$ in $L^{2}\left([0, T], L^{2}\left(\Sigma, \mathbb{R}^{n+2}\right)\right)$ and that the convolution and derivatives commute we obtain the result that $\mathbf{v}$ is an $L^{2}\left([0, T], L^{2}\left(\Sigma, \mathbb{R}^{n+2}\right)\right)$ function of the form $\left(\partial_{1} u, \ldots, \partial_{n} u, \partial_{t} u, u\right)$.

Collecting the results from this section we have established Theorem 2 ,

\section{Applications}

Although in the following three examples the spacetimes are not spatially compact we will assume we are working in a local region of the form $\Sigma_{[0, T]}=$ $[0, T] \times \Sigma$ as described in the geometric setting.

Junction Conditions There is a precise mathematical formalism proposed by Israel to describe the junction conditions for two regular spacetimes joined along a non-null singular hypersurface $\Lambda$ [7]. He noted that if we consider two half-spaces $V^{+}$and $V^{-}$, a singular hypersurface, $\Lambda$, can be fully characterised by the different extrinsic curvatures (second fundamental forms) associated with its embeddings in $V^{+}$and $V^{-}$and a continuous matching condition of the metric through the common boundary. If we use Gaussian coordinates based on $\Lambda$, then the normal derivatives of the metric have a jump across $\Lambda$ with the metric being continuous along $\Lambda$. This scenario satisfies the analytic conditions required for the application of Theorem 2 to apply. Notice however that the theorem does not need to make assumptions on the time dependence or matter content of the spacetime.

Impulsive Gravitational Waves A spacetime that contains impulsive gravitational waves described in double null coordinates has line element given by:

$$
d s^{2}=2 d u d v-(1-u \Theta(u))^{2} d y^{2}-(1+u \Theta(u))^{2} d z^{2}
$$


where $\Theta(u)$ is the Heaviside step function. The spacetime is vacuum, but has a Weyl tensor with delta function components

$$
\begin{aligned}
& C_{u y u y}=-\delta(u) \\
& C_{u z u z}=\delta(u)
\end{aligned}
$$

It is important to notice that although the curvature is not bounded this condition is not relevant for Theorem 2 to apply.

Brane-world Cosmologies The Randall-Sundrum Brane-Worlds (RS) are models that explore gravity beyond classical general relativity [31, and also appear in a cosmological context 32. In the RS model in $A d S_{5}$ one has that in Gaussian normal coordinates $X^{A}=\left(t, x^{i}, y\right)$ based on the brane at $y=0$, the model has the line element

$$
d s^{2}=e^{-2|y| / L}\left(-d t^{2}+d x^{i 2}\right)+d y^{2}
$$

This spacetime again satisfies the conditions for the Theorem 2 to be applicable and therefore solutions with finite energy exist.

Notice that the well-posedness result can be extended to other brane world models and even collision of branes as long as the spacetime satisfies the assumptions of Theorem 2. Therefore, one can consider that dynamical models of colliding branes (see e.g. [33]) do not produce strong gravitational singularities provided that the spacetime remains $C^{0,1}$ during all the process.

\subsection{Main result and the relation to previous work}

The main result of the paper (Theorem 2) shows that spacetimes which are usually thought of as singular may be regarded as regular if one adopts the point of view that true singularities make the local dynamics of test fields ill-defined. We have established general conditions under which linear wave equations are locally well-posed in spacetimes with weak singularities where the singularity is concentrated on a submanifold. In particular, the results can be applied to spacetimes with shell-crossing singularities, surface layers and hypersurface singularities of regularity $C^{0,1}$.

We establish local well-posedness for general first order linear symmetric hyperbolic systems with coefficients with low regularity. We show that unique stable solutions exist in $L^{2}\left(0, T ; L^{2}\left(\Sigma, \mathbb{R}^{N}\right)\right)$. This solution corresponds in the second order formalism to a finite energy solution in $H^{1}$ of the wave equation. Moreover, the main advantage in writing the problem as a first order system is that the existence of a covariantly constant timelike vector fields and the condition on the curvature are not needed which are key conditions in previous works [34, 18]. Therefore, the results obtained extend previous results of Vickers and Wilson [18] and Ishibashi and Hosoya [14] by allowing a larger class of non-vacuum time dependant spacetimes. We also 
establish not only the existence and uniqueness of solutions but also their stability and local well posedness.

\section{Acknowledgements}

The authors would like to thank CONACyT for supporting this work through a CONACyT Graduate Fellowship.

\section{References}

[1] J. Leray "Hyperbolic Differential Equations", Institute for Advanced Study, (1953)

[2] A. Bernal, M. Sanchez Class. Quantum Grav. 247452007

[3] S.W. Hawking, G.F.R. Ellis, The large scale structure of spacetime Cambridge University Press, (1974).

[4] M. Kunzinger, R. Steinbauer, M. Stojkovic, J. Vickers Class. Quantum Grav. 320750122015

[5] P.T. Chruściel, J.D.E. Grant Class. Quantum Grav. 291450012012

[6] C. J. S. Clarke Class. Quantum Grav. 159751998

[7] W. Israel Il Nuovo Cimento B Series 1011 Luglio, Volume 44, Issue 1, 1-14 1966

[8] R. Penrose The geometry of impulsive gravitational waves. In L. O'Raifeartaigh, editor, General Relativity, pages 101-115. Clarendon Press, (1972).

[9] C. J. S. Clark and N O'Donnell Rendiconti del Seminario Matematico, Universitá Torino 50391992

[10] I. Rácz Class. Quantum Grav. 271550072010

[11] R. Wald J Math Phys. 21, 28201980

[12] G. Horowitz, D. Marolf Phy. Rev. D 52, 56701995

[13] B.S. Kay, U.M. Studer Communications in Mathematical Physics 139, 1031991

[14] A. Ishibashi, A. Hosoya Phy. Rev. D 60, 1040281999

[15] Y. Sanchez Sanchez, J. A. Vickers Class.Quant.Grav. 332050022016 
[16] J.D.E. Grant, E. Mayerhofer, and R. Steinbauer, Comm. Math. Phys. 2853992009

[17] L.C. Evans Partial Differential Equations American Mathematical Society, (2002).

[18] J. A. Vickers, J. P. Wilson ArXiv:0101018 2001

[19] H. Ringström The Cauchy Problem in General Relativity ESI Lectures in Mathematics and Physics (2009)

[20] P. Szekeres, A. Lun J. Austral. Math. Soc. Ser. B 41, 167-179 1999

[21] R. Geroch, J. Traschen Phys. Rev. D 36, 10171987

[22] R. Steinbauer, J.A.G Vickers Class. Quantum Grav. 2662008

[23] A. Almheiri, D. Marolf, J. Polchinski, J. Sully Journal of High Energy Physics 2622013

[24] Y. Sanchez Sanchez General Relativity and Gravitation 47802015

[25] K.O. Friedrichs Comm. Pure Appl. Math. 721954

[26] J.P. Nicolas, C. R. Acad. Sci. Paris, Ser. 13442007.

[27] F. Colombini, E. De Giorgi, S. Spagnolo Ann. della Scuola Normale Superiore di Pisa 631979

[28] F. Colombini, N. Lerner Duke Math. Journal 7731995

[29] F. Colombini, G. Métivier ArXiv:0611426

[30] C. Garetto, M. Ruzhansky Archive for Rational Mechanics and Analysis 21712015

[31] T. Shiromizu, K. Maeda, M. Sasaki, Phys. Rev. D 62, 0240122000

[32] A.Hebecker, J. March-Russell Nucl. Phys. B 608 375-393 2001

[33] J. Lehners, P. Madden, and N. Turok Phys. Rev. D 75, 1035102007

[34] J. P. Wilson Class. Quantum Grav. 1731992000 